\title{
Article
}

\section{Self-reported psychopathy and aggression motivation: A role for emotions?}

Ireland, Jane Louise, Lewis, Michael, Ireland, Carol Ann, Derefaka, Gail, Chu, Simon and Archer, John

Available at http://clok.uclan.ac.uk/31075/

Ireland, Jane Louise ORCID: 0000-0002-5117-5930, Lewis, Michael ORCID: 0000-0001-5567-3569, Ireland, Carol Ann ORCID: 0000-0001-7310-2903, Derefaka, Gail, Chu, Simon ORCID: 0000-0001-8921-4942 and Archer, John ORCID: 0000-0003-0483-1576 (2020) Self-reported psychopathy and aggression motivation: A role for emotions? Journal of Forensic Psychiatry and Psychology, 31 (1). pp. 156-181. ISSN 1478-9949

It is advisable to refer to the publisher's version if you intend to cite from the work. http://dx.doi.org/10.1080/14789949.2019.1705376

For more information about UCLan's research in this area go to

http://www.uclan.ac.uk/researchgroups/ and search for <name of research Group>.

For information about Research generally at UCLan please go to http://www.uclan.ac.uk/research/

All outputs in CLoK are protected by Intellectual Property Rights law, including Copyright law. Copyright, IPR and Moral Rights for the works on this site are retained by the individual authors and/or other copyright owners. Terms and conditions for use of this material are defined in the policies page.

\section{CLoK}

Central Lancashire online Knowledge www.clok.uclan.ac.uk

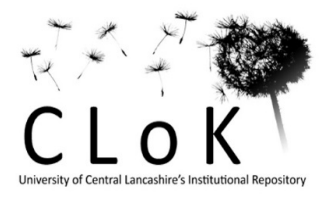


RUNNING HEAD: PSYCHOPATHY, AGGRESSION MOTIVATION AND EMOTION

Self-reported psychopathy and aggression motivation: A role for emotions?

Jane L. Ireland ${ }^{1 *}$, Michael Lewis*, Carol A Ireland*, Gail Derefaka, Louise Taylor, Joanne McBoyle, Louise Smillie, Simon Chu*, John Archer

University of Central Lancashire, Preston, UK; *and Ashworth Research Centre, Mersey

Care NHS Trust: UK.

${ }^{1}$ Corresponding author. Email: JLIreland1@uclan.ac.uk; University of Central Lancashire, Preston, UK, PR1 2HE; Tel: +44 1772201201. 


\begin{abstract}
The current study examined the psychopathy-aggression relationship by considering different forms of aggression, including aggressive motivation. Emotions were explored as useful in understanding any emerging relationship, notably those consistent with detachment and sensitivity/poor regulation. In three studies, involving non-offending samples (Study one: $n=$ 150, 47 men, 103 women; Study two: $n=442,314$ men, 123 women, five not disclosed; Study three: $n=200,100$ men and 100 women), it was predicted that higher levels of psychopathy would be associated with reports of higher overall, proactive and reactive aggression, and that the association would be moderated by emotion. Specifically, it was predicted that emotion regulation difficulties would associate with higher levels of reactive but not proactive aggression, and that proactive aggression would involve a lack of emotion. Findings generally supported the predictions. The role of emotion-regulation difficulties as a mediator between psychopathy and reactive aggression, and greater emotional detachment as a mediator between psychopathy and proactive aggression was demonstrated. The results demonstrate importance for the components of psychopathy and different aspects of emotion for the psychopathyaggression link. Directions for future research and some implications for practice are outlined.
\end{abstract}


Self-reported psychopathy and aggression motivation: A role for emotions?

Psychopathy involves maladaptive personality traits comprising interpersonal, affective, behavioural and dissocial features, with the interpersonal and affective components relating to Factor I psychopathy and dissocial features to Factor II (Hare, 2003). An important distinction is between primary and secondary psychopathy. The former demonstrates a predisposition towards interpersonal and affective components of psychopathy, notably a lack of prosocial emotions. Secondary psychopathy is characterised more by affect dysregulation, hostility, and emotional blunting associated with childhood trauma (Blackburn, 1998; Porter, 1996). Regardless of the origins of psychopathy, there is consensus that it comprises subtypes, and that psychopathy is associated with several forms of dissocial behaviour, including aggression. The association between aggression and these psychopathy subtypes has been less extensively researched than that between aggression and the global psychopathy concept, despite recognition of the heterogeneity of psychopathy.

A core reason as to why psychopathy has been of interest to both academics and clinicians, represents its relationship with aggression. An association between psychopathy and aggression has been long established (e.g. Asscher et al., 2011; Guy, Douglas \& Hendry, 2010; Walters, 2003), with a greater degree of psychopathy being associated, at least moderately, with higher levels of aggression frequency (Blais, Solodukhin \& Forth, 2014). However, the attention given to non-forensic samples is sparse, with that afforded to aggression motivation equally limited. The latter is surprising considering an increased acceptance in aggression research of a need to capture motivation when trying to formulate the causes and facilitators of aggression (Ohlsson \& Ireland, 2011).

Aggressive motivation is most commonly viewed as either reactive, which involves an immediate emotionally-driven response to frustration or provocation, or proactive, which is 
controlled and goal-oriented (Vitaro \& Brendgen, 2005). It has been suggested that psychopathy is more closely associated with proactive than with reactive aggression (Porter \& Woodworth, 2006). This has been confirmed in some studies (e.g. Cornell et al., 1996; Raine, Fung, Portnoy, Choy \& Spring, 2014; Vitacco, Neumann \& Caldwell, 2010; Walsh, Swogger \& Kosson, 2009). However, research also suggests that it is the Factor I (interpersonal/affective) component of psychopathy, rather than Factor II (Dissocial behaviour/lifestyle), that is closely linked with proactive aggression (Bezdjian, Tuvblad, Raine \& Baker, 2011). Indeed, a subsequent meta-analysis of Blais et al. (2014), found moderate associations between overall psychopathy and higher levels of both proactive and reactive aggression. This link was found to be stronger for self-report scales, than clinical assessments.

Although there is an emerging conclusion that psychopathy is linked to both reactive and proactive aggression, research in this area is limited in terms of examining the variables that could moderate the aggression-psychopathy relationship. A role for emotions appears a logical consideration (e.g. Long, Felton, Lilienfeld \& Lejuez, 2014; Roberton, Daffern \& Bucks, 2012), since reactive and proactive aggression are generally distinguished on the basis of their emotional content; proactive involving the absence of emotion, and, reactive, the presence of unmanaged emotion. Those with higher levels of proactive aggression have, for example, a reduced responsiveness to distressing stimuli, thus suggesting that there is more emotional poverty among those who display such aggression (Kimonis, Frick, Fazekas \& Loney, 2006). In addition, there has been increasing interest in the role of emotional functioning in psychopathy among those with a violent history (e.g. Garofalo, Neumann \& Velotti, 2018). Garofalo et al (2018), for example, argued emotional dysregulation as a core feature in their sample of violent male offenders, noting how it involved broad challenges across the domain of emotional regulation. They noted how higher levels of psychopathy were related to greater levels of emotional dysregulation, particularly when considering lifestyle and 
affective psychopathic traits, as opposed to interpersonal traits. Although this study did not examine non-violent offenders, or aggression motivation, what it did capture was the importance of considering emotional dysregulation alongside different psychopathic components, thereby moving away from viewing psychopathy as a heterogeneous concept. In doing so, they were able to demonstrate an association between emotional dysregulation and the more affective/secondary aspects of psychopathy.

An association between psychopathy and violence is also expected through a theoretical lens; the Violence Inhibition Mechanism model (VIM; Blair, 1995, 2001) proposes that individuals learn to modulate aggression through perceiving expressions of distress in others (Fido, Santo, Bloxsom, Gregson \& Sumich, 2017). In its simplest form, the VIM holds that during early socialisation individuals develop representations of context through perspectivetaking (i.e. via classical-conditioning), which is a form of empathy. Dysfunction of this system is implicated in the development of psychopathy (e.g. through callous and unemotional traits forming: Fido et al., 2017), and becomes manifest through an inability to empathise with others (Domes, Hollerbach, Vohs, Mokros \& Habermeyer, 2013). The apparent consensus that psychopathy is a product of deficits in processing negative emotional stimuli is a largely convincing one (Blair, Morris, Frith, Perrett \& Dolan, 1999; Loney, Frick, Clements, Ellis \& Kerlin, 2003). It would be logical, therefore, to consider whether these recognised deficits with emotional responding could contribute to an explanation of the association between proactive and reactive aggression, and psychopathy. There are advantages in considering this with a non-offending sample to ensure that what is being assessed is the role of psychopathy and not anti-social behaviour per se, which may contaminate samples of offenders (Kimonis et al., 2006).

Research designed to understand how emotions could mediate any association between psychopathy and aggressive motivation is largely absent, with focus instead on basic 
descriptive factors, such as age and sex (Blais et al., 2014). This is not unique to the aggressionpsychopathy relationship, with researchers criticising the area for their lack of consideration of the range of emotions relevant to psychopathy. For example, Garofalo, Neumann, Zeigler-Hill \& Meloy (2019) argue for greater attention to a range of emotions when considering psychopathy and include spitefulness and contempt as core emotions to consider, with both associating with increased levels of psychopathy in non-forensic samples.

There has, nevertheless, been some attempt to consider emotion as a potential predictor of group membership in terms of aggressive motivation. A recent study of aggression in primary school children, who differed on psychopathic traits, found that those with both high and reactive aggression were more likely to show high callous-unemotional traits than the solely reactive and low aggression groups, with the reactive group being more likely to display deficits in planning, and inhibitory control (Thomson \& Centifanti, 2018). Using a sample of adult substance users, Long et al. (2014) specifically examined how emotions could mediate the association between psychopathy and aggression subtypes, in this case premeditated [proactive] and impulsive [reactive] aggression. They examined fearless dominance, selfcentred impulsivity and cold-heartedness, all factors of psychopathy. They found that fearlessness was related to premeditated but not impulsive aggression, self-centred impulsivity to both aggression types, with but cold-heartedness being related to either. Of particular interest, was the finding that the self-centred impulsivity and fearlessness components of psychopathy had an indirect effect on impulsive aggression via emotion regulation difficulties, although emotion regulation difficulties did not mediate the relationship between psychopathy and premeditated aggression. The overall conclusion was that emotional regulation challenges mediated the relationship between psychopathy and impulsive but not premeditated aggression.

The current study aims to further explore how emotions are associated with psychopathy and aggression motivation. It does so via a series of studies to allow for detailed 
examination. The first examines the association between trait aggression and psychopathy in a non-offender population, specifically investigating how the emotional components of psychopathy might be differentially related to aggression. Studies 2 and 3 progress to a detailed examination of aggressive motivation, and its association with both lack of emotion and poor emotion regulation. Different self-report psychopathy measures were used to explore whether there was consistency between measures, to test the generalisability of the psychopathyaggression link, and to study the link with reactive and proactive aggression motivation. Using different measures further allows for examination of psychopathy factors and primary/secondary psychopathy, which requires the application of differing self-report psychopathy measures, as no single measure captures both factors and the primary/secondary distinction. Adopting an approach whereby a multi-study employs different measures of psychopathy across studies is not novel (e.g. Garofalo et al, 2019). It is also important to note that whereas different measures were used to measure psychopathy, the overall character traits and agreement on the concept of psychopathy remains unaltered, with considerable consensus in the literature in relation to its properties (DeLisi, 2009, 2016). The collection of current studies also used a self-report of aggressive motivation and measures of emotions, employing both general and student samples, with the following core predictions:

1.) Higher levels of psychopathy will be associated with higher levels of overall aggression, and both proactive and reactive aggressive motivation (Blais et al., 2014).

2.) Association between psychopathy and aggressive motivation will be moderated by emotion regulation, with regulation difficulties associated with higher levels of reactive but not proactive aggression (e.g. Garofalo et al, 2018; Long et al., 2014).

3.) Association between psychopathy and proactive aggression will be moderated by a lack of emotion (e.g. Kimonis et al., 2006). 


\section{Study one: Psychopathy and trait aggression - exploring component differences}

Study 1 explored the link between the factors of psychopathy and their association with aggression and its factors. In doing so, it concerns itself with the first prediction. It represents the grounding study in terms of examining a link between psychopathy traits and general aggression.

\section{Method}

\section{Participants}

There were 150 participants from the general population (47 men and 103 women); 35 identified as students. The average age was $30.7(\mathrm{SD}=11.6)$.

\section{Procedure}

The study was conducted online and advertised via social media websites (e.g. Facebook, Twitter). The measures were preceded by a participant information sheet informing participants of the nature of the study and their right to withdraw. On completion of the survey, participants were directed to a debriefing sheet. Participants were offered no reward for their participation. Anonymity was ensured. Ethical approval was obtained from the University of Central Lancashire ethics committee.

\section{Measures}

The following measures were completed. Their psychometric properties can be found in Table 1.

Psychopathic Processing and Personality Assessment (PAPA, Lewis, Ireland, Abbott \& Ireland, 2017), is a self-report measure of psychopathy that was developed following a literature review, expert Delphi, interviews with forensic patients and non-patients, and a quantitative study with prisoners and students (Lewis, 2014). It is receiving increased recognition (Thomson, 2019), with a number of studies ongoing (e.g. Ireland et al, under 
review; Lewis et al, under review). The PAPA comprises 29 items and requires participants to rate how well a series of statements describes how they have been throughout their life. Example items include, "I am only interested in myself" and "I am not bothered about others". It is measured on a five-point Likert scale (' 1 ' being 'very unlike me' and ' 5 ' being 'very like me'). There are four subscales: (1) Dissocial; (2) Emotional detachment; (3) Disregard for others; and (4) Sensitivity to negative emotion.

Aggression Questionnaire (AQ: Buss and Perry, 1992). This measures trait aggression, which Buss and Perry (1992) described as having four components; physical aggression (e.g., "I have threatened people I know"), verbal aggression (e.g., "I tell my friends openly when I disagree with them"), anger (e.g., "I have trouble controlling my temper"), and hostility (e.g., "I sometimes feel people are laughing at me behind my back"). Items are scored on a Likert scale where ' 1 ' is extremely unlike me and ' 5 ' is extremely like me.

\section{Data Analytic Plan}

Descriptive statistics and internal consistency $\alpha$ coefficients were computed for all study variables. Bivariate correlations were initially conducted between the core variables of interest (psychopathy and aggression), to determine if a relationship existed, which could then be further explored. Once demonstrated, there was examination of the relationship in more detail via a series of linear regressions, to determine the contribution of psychopathy to increased levels of aggression. All analyses used the Enter command so predictors were entered without a predetermination of their likely relevance. This reflected the lack of research sufficient to confirm a specific variable ordering. In these analyses, psychopathy was entered as a potential predictor of overall aggression, and its subtypes. This was then replicated by examination of the factors of psychopathy as potential predictors of aggression. This allowed for initial consideration of the potential contribution of the emotional component of psychopathy and 
aggression types. Regressions were completed overall and for each AQ subscale, with total PAPA and PAPA subscales considered in separate analyses to avoid multicollinearity.

\section{Results}

Descriptive statistics for all variables are shown in Table 1.

$<$ Insert Table 1 here >

Total PAPA scores were highly positively correlated with total trait aggression on the AQ ( $r=$ $.78, p<.0001$ ), and for all AQ subscales (Physical, $r=.86, p<.0001$; Verbal, $r=.69, p<.0001$; Anger, $r=.86, p<.0001$; Hostility, $r=.77, p<.0001)$. PAPA Dissocial positively correlated with the total AQ $(r=.66, p<.0001)$ and all the AQ subscales (Physical, $r=.63, p<.0001$; Verbal, $r=.53, p<.0001$; Anger, $r=.50, p<.0001$; Hostility, $r=.46, p<.0001)$. PAPA Emotional detachment positively correlated with the total AQ $(r=.29, p<.0001)$ and all AQ subscales (Physical, $r=.28, p<.001$; Verbal, $r=.19, p<.02$; Anger, $r=.19, p<.02$; Hostility, $r=.25, p<.02)$. PAPA Disregard positively correlated with total AQ $(r=.59, p<.0001)$ and all AQ subscales (Physical, $r=.60, p<.0001$; Verbal, $r=.47, p<.0001$; Anger, $r=.47, p<$ .0001 ; Hostility, $r=.35, p<.0001)$. PAPA Sensitivity to negative emotion positively correlated with total AQ $(r=.78, p<.0001)$ and all AQ subscales (Physical, $r=.66, p<.0001$; Verbal, $r$ $=.50, p<.0001$; Anger, $r=.68, p<.0001 ;$ Hostility, $r=.64, p<.0001)$.

A series of standard multiple regression analyses were performed. The model considering total trait aggression and total psychopathy was significant $(\mathrm{F}(1,148)=192.6, p$ $<.0001)$, with higher levels of psychopathy predicting higher overall trait aggression $(\mathrm{t}=13.8$, $p<.0001, \mathrm{~B}=1.02, \mathrm{SE}=.07, \beta=.75)$ and accounting for $56 \%$ of the variance $\left(\mathrm{R}^{2}=.56\right.$, Adjusted $\mathrm{R}^{2}=.56$ ). Total psychopathy also predicted higher levels of physical aggression $($ Overall model $(\mathrm{F}(1,148)=154.7, p<.0001$; Predictor, $\mathrm{t}=12.4 p<.0001, \mathrm{~B}=11.3, \mathrm{SE}=.91$, 
$\beta=.71$; predicting $51 \%$ of the variance $\left(\mathrm{R}^{2}=.51\right.$, Adjusted $\left.\mathrm{R}^{2}=.50\right)$, verbal aggression $($ Overall model $(\mathrm{F}(1,148)=72.1, p<.0001 ;$ Predictor, $\mathrm{t}=8.49 p<.0001, \mathrm{~B}=3.94, \mathrm{SE}=.46, \beta$ $=.57$; predicting $32 \%$ of the variance $\left(\mathrm{R}^{2}=.32\right.$, Adjusted $\left.\mathrm{R}^{2}=.32\right)$, trait anger $($ Overall model $(\mathrm{F}(1,148)=92.9, p<.0001 ;$ Predictor, $\mathrm{t}=9.64 p<.0001, \mathrm{~B}=8.30, \mathrm{SE}=.86, \beta=.62 ;$ predicting $38 \%$ of the variance $\left(\mathrm{R}^{2}=.38\right.$, Adjusted $\left.\mathrm{R}^{2}=.38\right)$ and hostile aggression (Overall model $(\mathrm{F}(1,148)=71.6, p<.0001 ;$ Predictor, $\mathrm{t}=8.46 p<.0001, \mathrm{~B}=7.94, \mathrm{SE}=.94, \beta=.57$; predicting $32 \%$ of the variance $\left(\mathrm{R}^{2}=.32\right.$, Adjusted $\left.\mathrm{R}^{2}=.32\right)$.

The model considering total trait aggression and the four factors of psychopathy was also significant $(\mathrm{F}(4,145)=76.2, p<.0001)$, with higher levels of the dissocial psychopathy component $(\mathrm{t}=5.39, p<.0001, \mathrm{~B}=.04, \mathrm{SE}=.008, \beta=.33)$ and sensitivity to emotional regulation $(\mathrm{t}=9.78 p<.0001, \mathrm{~B}=.08, \mathrm{SE}=.008, \beta=.60)$ predicting higher levels of trait aggression, and accounting for $67 \%$ of the variance $\left(\mathrm{R}^{2}=.67\right.$, Adjusted $\left.\mathrm{R}^{2}=.66\right)$. Emotional detachment and disregard components were not predictors $(\mathrm{t}=-1.35$ and .36 respectively).

The model considering physical aggression and the four factors of psychopathy was also significant $(\mathrm{F}(4,145)=47.5, p<.0001)$, with higher levels of the dissocial psychopathy component $(\mathrm{t}=4.40, p<.0001, \mathrm{~B}=.47, \mathrm{SE}=.11, \beta=.31)$ and sensitivity to emotional regulation $(\mathrm{t}=5.59 p<.0001, \mathrm{~B}=.62, \mathrm{SE}=.11, \beta=.40)$ predicting higher physical aggression and accounting for $57 \%$ of the variance $\left(\mathrm{R}^{2}=.57\right.$, Adjusted $\left.\mathrm{R}^{2}=.55\right)$. Emotional detachment was also a predictor $(\mathrm{t}=2.49 p<.014, \mathrm{~B}=.33, \mathrm{SE}=.13, \beta=.20)$ whereas the disregard component was not $(\mathrm{t}=-1.09)$.

The model considering verbal aggression and the four factors of psychopathy was also significant $(\mathrm{F}(4,145)=20.7, p<.0001)$, with higher levels of the dissocial psychopathy component $(\mathrm{t}=3.66, p<.0001, \mathrm{~B}=.21, \mathrm{SE}=.06, \beta=.32)$ and sensitivity to emotional regulation $(\mathrm{t}=3.09 p<.002, \mathrm{~B}=.18, \mathrm{SE}=.06, \beta=.27)$ predicting higher verbal aggression 
and accounting for $36 \%$ of the variance $\left(\mathrm{R}^{2}=.36\right.$, Adjusted $\left.\mathrm{R}^{2}=.34\right)$. Emotional detachment and disregard components were not predictors $(\mathrm{t}=-1.11$ and 1.64 respectively).

The model considering trait anger and the four factors of psychopathy was also significant $(\mathrm{F}(4,145)=35.4, p<.0001)$, with higher levels of the dissocial psychopathy component $(\mathrm{t}=2.41, p<.017, \mathrm{~B}=.24, \mathrm{SE}=.09, \beta=.19)$ and sensitivity to emotional regulation $(\mathrm{t}=7.54 p<.0001, \mathrm{~B}=.76, \mathrm{SE}=.10, \beta=.59)$ predicting higher trait anger and accounting for $49 \%$ of the variance $\left(\mathrm{R}^{2}=.49\right.$, Adjusted $\left.\mathrm{R}^{2}=.48\right)$. Emotional detachment and disregard components were not predictors $(\mathrm{t}=-1.66$ and 1.63 respectively).

The model considering hostile aggression and the four factors of psychopathy was also significant $(\mathrm{F}(4,145)=29.8, p<.0001)$, with higher levels of the dissocial psychopathy component $(\mathrm{t}=3.00, p<.003, \mathrm{~B}=.32, \mathrm{SE}=.11, \beta=.24)$ and sensitivity to emotional regulation $(\mathrm{t}=7.62 p<.0001, \mathrm{~B}=.83, \mathrm{SE}=.11, \beta=.62)$ predicting higher verbal aggression and accounting for $45 \%$ of the variance $\left(\mathrm{R}^{2}=.45\right.$, Adjusted $\left.\mathrm{R}^{2}=.44\right)$. Disregard was also a predictor $(\mathrm{t}=-2.09 p<.04, \mathrm{~B}=-.28, \mathrm{SE}=.13, \beta=-.19)$, with lower levels of disregard predicting higher levels of hostility. The emotional detachment component was not a predictor $(\mathrm{t}=.49)$.

\section{Discussion}

Higher levels of psychopathy were associated with higher levels of trait aggression. This applied to all four subscales, which is broadly consistent with prior research (Asscher et al., 2011, Blais et al., 2014; Guy et al., 2010; Walters, 2003). There were notable differences between psychopathy factors in the strength of this relationship: these appeared to be associated with the emotional elements of the construct, in that the emotional detachment factor of psychopathy showed only a low association with overall trait aggression, and its subscales. The strongest correlations were found with the sensitivity to negative emotion factor, with higher levels of this associated with higher levels of total aggression and all subscales. This provides some potential support for previous findings indicating the role of emotional 
dysregulation in explaining the psychopathy-aggression relationship in terms of impulsive aggression (Long et al., 2014). The present findings suggest that this could be extended to trait aggression overall and to its sub-components. Overall, there was evidence of differences in the pattern of associations between psychopathy and aggression, consistent with prior research (Long et al., 2014). Although total psychopathy predicted total trait AQ, and across the subscales, this was not consistent across the factors of psychopathy, with factors representing dissocial and sensitivity to negative emotion predicting higher levels of aggression. Indeed, the individual factors of psychopathy were explaining more of the variance overall, with the only exception representing overall psychopathy and physical aggression. Thus, it could be speculated that psychopathy was predicting increased physical aggression primarily, but differences are emerging when considering the components of aggression and the individual components of psychopathy. The emotional components of psychopathy had different strengths of association with aggression measures, with sensitivity to negative emotions showing a close association with aggression, whereas emotional detachment showed only a weak association. This is of particular interest since the former is more closely associated with secondary psychopathy and the latter with primary psychopathy; it would suggest that it is the emotional component of secondary psychopathy that is more closely associated with aggression.

\section{Study 2: Psychopathy and aggression motivation: Is emotional regulation important?}

Study 2 concerns itself primarily with prediction two, focusing on emotional regulation as a factor that could contribute to an understanding of the psychopathy-aggression relationship. It applies a different measure of psychopathy to assist with determining the generalisation of 
findings and contributing further to an examination of finding consistency in relation to prediction 1, using an alternative measure.

\section{Method}

\section{Participants}

A total of 442 participants completed the study, $71 \%(n=314)$ were men, $27.8 \%(n=123)$ were women, and $1.1 \%(\mathrm{n}=5)$ identified as having no preferred sex or as transgendered. Participants' ages ranged from 18 to $66(\mathrm{M}=25.6, \mathrm{SD}=8.0)$.

\section{Procedure}

Ethical approval was obtained from the university. The study was conducted online, and participants were mostly recruited via social media. Details of the study were posted on sites including Facebook, Twitter and LinkedIn, and posters were also displayed at the university campus. Participants were informed their participation would be anonymous. On completion of the study, participants were debriefed via an online debrief sheet.

\section{Measures}

The following measures were completed. Their psychometric properties can be found in Table 2.

Short Dark Triad (SD3; Jones \& Paulhus, 2014), a 27 item questionnaire used to measure levels of sub-clinical narcissism, psychopathy and Machiavellianism. Each subscale contained nine questions and answers were on a 5 point Likert scale, ranging from ' 1 ' disagree strongly to ' 5 ' agree strongly. The psychopathy component was focused on for the current research, with an example item being, "I like to get revenge on authorities".

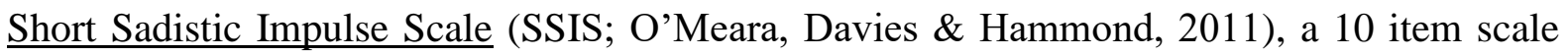
used to measure sadistic tendencies. Items were presented in a dichotomous format and participants were asked to rate each item as 'like me' or 'unlike me'. Examples of questions included, "I enjoy seeing people hurt" and "I have humiliated others to keep them in line." 
Reactive-Proactive Aggression Questionnaire (RPQ; Raine et al., 2006) comprises 23 items and was used to assess levels of proactive and reactive aggression motivations. It was originally used in adolescent samples, although has since been used with adults (Archer \& Thanzami, 2009; Brugman et al., 2017; Cima, Raine, Meesters, \& Popma, 2013). Items include, "Had fights with others to show who was on top." (proactive) and, "Had temper tantrums" (reactive). Participants were asked to rate how often they had experienced each item on a scale of ' 0 ' (never), '1' (sometimes), or '2' (often). The wording was adjusted to fit an adult sample (e.g. any references to other 'students' were altered to other 'people').

Difficulties in Emotion Regulation Scale (DERS; Gratz \& Roemer, 2004), a 36-item scale used to measure emotion dysregulation. Participants were asked to rate how often they experienced difficulties with accepting, recognising, controlling and regulating emotions, as well as how often they felt unable to engage in goal-directed behaviour due to their emotions. Answers were on a 5-point scale, ranging from ' 1 ' almost never to '5' almost always. Statements included, "When I'm upset, I feel guilty for feeling that way," and "When I'm upset, I become out of control."

\section{Data Analytic Plan}

Descriptive statistics and internal consistency $\alpha$ coefficients were computed for all study variables. Building on Study 1, which showed how aggression type and emotional factors were of interest, bivariate correlations were here computed between psychopathy and, 1.) the different forms of aggression (reactive/proactive); and 2.) specific emotional factors, which comprised the absence of emotion (sadism) and that of emotional dysregulation. Having further demonstrated a relationship between psychopathy and aggression, a series of simple mediation analysis were then conducted to explore whether the emotional factors mediated this relationship. These were completed using a series of multiple regressions and using the Sobel 
Test to determine significance of any resulting indirect effects. In all analyses, the criterion variable represented either reactive or proactive aggression.

\section{Results}

Descriptive statistics for all variables are shown in Table 2.

< Insert Table 2 here >

Significant positive correlations were found between total psychopathy and higher levels of proactive $(r=.49, p<.01)$ and reactive $(r=.65, p<.01)$ aggression, and higher levels of sadism $(r=.57, p<.01)$ and emotional dysregulation $(r=.23, p=<.01)$. Emotional dysregulation and sadism were also positively correlated with reactive $(r=.46, p<.01$ and $r=.52, p<.01$ respectively) and with proactive aggression $(r=.23, p<.01$ and $r=.62, p<.01$ respectively). The simple mediation analysis were then conducted. The results are presented in Figures 1 and 2.

Higher emotional dysregulation was found to be a partial mediator of the association between higher psychopathy and higher reactive aggression but was not a mediator in the case of proactive aggression. A higher level of sadism was a partial mediator of the relationship between higher psychopathy and both greater reactive and proactive aggression. Partial mediations were all at the $p<.0001$ level, using the Sobel Test.

< Insert Figures 1 and 2 here >

\section{Discussion}

Although the correlations between psychopathy and aggression were more modest in this study than the prior study, it remained the case that higher levels of psychopathy were associated 
with both more reactive and proactive aggression. It was also associated with sadism and emotional dysregulation, which is consistent with previous research (Asscher et al., 2011; Blais et al., 2014; Guy et al., 2010; Garofalo et al, 2018). Such findings also provide evidence of construct validity for the SD3 psychopathy subscale and emphasise the benefits of adopting self-report to examine the construct in non-forensic samples.

Furthermore, higher levels of emotional dysregulation and sadism correlated with greater proactive and reactive aggression. The correlation between sadism and proactive aggression was stronger than that between sadism and reactive aggression, whereas emotional dysregulation related better to reactive aggression. Higher levels of sadism acted as a partial mediator between psychopathy and higher levels of both proactive and reactive aggression, indicating that sadism is performing with some similarity across both aggression motivations. Emotional dysregulation partially mediated the association between psychopathy and reactive, but not proactive, aggression, which indicates a role for emotional sensitivity/poor regulation in facilitating emotionally-driven aggression and is characteristic of Factor II or secondary psychopathy (Bezdjian et al., 2011; Garofalo et al, 2018).

Whilst there appears a role for emotion and its regulation in understanding the psychopathy-aggression relationship, its impact remains unclear. It could be speculated that the findings convey the benefits of conceptualising emotions on a continuum, where absence of concern or care appear at one end (i.e. emotional detachment) and hypersensitivity to/poor regulation of emotion at the other (i.e. emotional sensitivity/poor regulation). For reactive aggression, it would appear this is likely to involve emotional sensitivity/poor regulation and for proactive aggression, emotional detachment. 


\section{Study 3: Unemotional traits: How do these moderate the psychopathy and aggression motivation relationship?}

Study 3 further explores emotional functioning and aggression, but in this instance does so by investigating the link between further components of psychopathy, namely primary and secondary. It used the same measure of aggression motivation from Study 2, in order to provide consistency in the aggression variable. It utilised a method of psychopathy that was able to capture primary and secondary psychopathy directly. The current study concerns itself primarily with prediction 3 but also provides some further reliability of the aggressionpsychopathy relationship with a further measure of psychopathy (prediction 1). It builds further on the findings from Study 2 by extending exploration of callousness/unemotionality, beyond sadism.

\section{Method}

\section{Participants}

Two hundred and four participants were recruited. The data from four were excluded as they did not complete all questionnaires. The remaining 200 participants were all undergraduate students, 100 men and 100 women $($ Mean age $=23.0, \mathrm{SD}=6.2$ ). Data from a further two participants were removed following data screening, resulting in a final total of 198.

\section{Procedure}

Ethical approval was obtained from the university ethics committee. Participants were approached in communal areas on the university campus. The information sheet explained that engagement was anonymous. It also contained instructions on how to complete and return the questionnaire packs. A secure box was provided for participants to return their completed packs. 


\section{Measures}

The following measures were completed. Their psychometric properties can be found in Table 3.

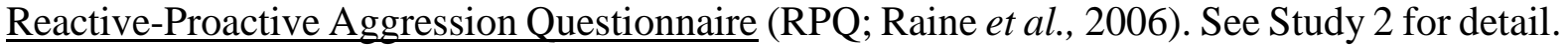
Levenson Self-Report Psychopathy Scale (LSRP; Levenson, Kiehl \& Fitzpatrick, 1995), which comprised 26 items divided into two subscales, primary psychopathy (e.g. "for me, what's right is whatever I can get away with"), and secondary psychopathy (e.g. "I find myself in the same kinds of trouble, time after time”). All items were rated on a 4-point scale: '0' disagree strongly to ' 3 ' agree strongly.

Inventory of Callous-Unemotional Traits (ICU; Frick, 2004), which comprised 23 items divided into three subscales. The first was callousness (11 eleven items, e.g. "I do not care who I hurt to get what I want"), the second, uncaring (eight items, e.g. "I care about how well I do at school or work") and the third, unemotionality (five items, e.g. "I do not show my emotions to others"). All items were rated on a four point scale ranging from ' 0 ' not at all true to ' 3 ' definitely true.

\section{Data Analytic Plan}

Descriptive statistics and internal consistency $\alpha$ coefficients were again computed for all study variables. Bivariate correlations were initially computed between psychopathy and aggression motivation to demonstrate a continued association, before proceeding to a series of simple mediation analyses to explore whether the ICU subscales of callousness, uncaring and unemotionality mediated the demonstrated relationship between psychopathy (total, primary and secondary) and either reactive or proactive aggression motivation. Reactive aggression was captured as part of these analyses owing to its evidenced contribution to the psychopathyaggression relationship in the prior study. There was no specific prediction made in relation to reactive and, consequently, it is exploratory. Analyses were completed using a series of 
multiple regressions and the Sobel Test to determine significance of any resulting indirect effect. In all analyses, the criterion variable represented either reactive or proactive aggression.

\section{Results}

Descriptive statistics for all the variables are shown in Table 3.

< Insert Table 3 here >

There was a moderate significant relationship between reactive aggression and psychopathy $(r$ $=.32, \mathrm{p}<.01)$, with weaker, but still significant, relationships with $\mathrm{CU}$ traits $(\mathrm{r}=.28, \mathrm{p}<.01)$. For proactive aggression, strong significant relationships were shown with both psychopathy $(r=.52, \mathrm{p}<.01)$ and CU traits $(r=.52, \mathrm{p}<.01)$.

A series of simple mediation analyses were subsequently conducted to explore whether the ICU subscales of callousness, uncaring and unemotionality mediated the relationship between psychopathy (total, primary and secondary) and either reactive or proactive aggression. Reactive aggression was captured as part of these analyses owing to its evidenced contribution to the psychopathy-aggression relationship in the prior study. There was no prediction made in relation to reactive and, consequently, it is exploratory.

Analyses were completed using a series of multiple regressions and using the Sobel Test to determine significance of any resulting indirect effect. In all analyses, the criterion variable represented either reactive or proactive aggression. The significant analyses are presented in Figures 3 to 5.

$<$ Insert Figure 3 here > 
Higher levels of callousness partially mediated the relationship between overall psychopathy and reactive aggression, with this holding for secondary psychopathy but not primary psychopathy. Higher callousness was also a partial mediator of the association between psychopathy (total, primary and secondary) and proactive aggression. However, higher callousness was not a mediator of the association between primary psychopathy and higher levels of reactive aggression.

$<$ Insert Figure 4 here >

Lower levels of caring partially mediated between primary psychopathy and reactive aggression and were also a partial mediator of the relationship between psychopathy (total, primary, secondary) and proactive aggressive.

$<$ Insert Figure 5 here >

Higher levels of unemotionality were also found to be a partial mediator between psychopathy (total, primary, secondary) and proactive aggression, but not reactive aggression. Partial mediations were all at the $p<.0001$ level, using the Sobel Test.

\section{Discussion}

Higher levels of callousness were found to be a partial mediator of the association between both overall psychopathy, and its components (primary and secondary psychopathy) and proactive aggression. Lack of caring was also a partial mediator of these relationships, with higher levels of unemotionality partially mediating the link between all factors of psychopathy and proactive aggression. 
The findings of Study 3 suggest that callous-unemotional traits correlate with, and mediate between, psychopathy and proactive aggression. However, all three emotions characteristic of emotional detachment (i.e. callousness, uncaring and unemotional) consistently mediated the link between psychopathy and proactive aggression. Thus, emotional detachment appears to be driving the psychopathy-proactive aggression relationship; aggression arising from this can therefore be assumed to be influenced by the emotional challenges often encountered with primary psychopathy or Factor I (Blackburn, 1998, Porter, 1996). In addition, there was an association with reactive aggression and psychopathy, which was mediated by callousness and a lack of care. This was an exploratory analysis but indicated, nevertheless, the importance of capturing reactive aggression.

\section{Overall discussion}

The findings from these three studies, using three different measures of psychopathy and two different aggression scales, demonstrate an association between psychopathy and aggression, primarily for physical aggression, when trait aggression is considered, and for reactive and proactive aggression, when aggression motivation was measured. This was consistent with prediction 1, and prior research (Asscher et al., 2011, Blais et al., 2014; Guy et al., 2010; Walters, 2003). It further confirms that psychopathy is not only associated with proactive aggression (Walsh et al., 2009; Vitacco et al., 2010). The current research further identifies emotions as core factors to consider but notes how their impact is far from uniform and seemingly determined by aggression motivation and the type of emotion under consideration. The studies collectively suggest that emotional detachment is a key consideration when accounting for proactive aggression (Kimonis et al., 2006), whereas emotional-sensitivity/poor regulation is of greater importance when considering reactive aggression (Bezdjian et al., 2011; Long et al., 2014). The predictions made were consequently supported, namely that the association between psychopathy and reactive (but not proactive) aggression would be 
moderated by emotional regulation difficulties, and the psychopathy-proactive relationship, by a lack of emotion. It is also consistent with the research of Garofalo et al (2018) who highlighted emotional dysregulation as a key consideration in understanding psychopathy, more so for that aligned to secondary psychopathy traits. The current study lends further support to the suggestion that emotional dysregulation is key more to the secondary psychopathy.

Emotions are, consequently, important factors of interest in the psychopathy-aggression relationship. In Study 1, we found that the psychopathy components associated with a lack of emotion (i.e. emotional detachment) were either unrelated to aggression, or had a limited association. However, psychopathy components linked with emotional-sensitivity and poor regulation were related to aggression. Although this finding suggests that Factor II psychopathic traits were dominating the psychopathy-aggression relationship, Studies 2 and 3 provided a more detailed analysis. They found that emotional dysregulation related better to reactive than to proactive aggression motivation, and that callous-unemotional traits mediated the association between psychopathy and proactive aggression. Study 1 also demonstrated that the emotional components of psychopathy raised questions about the roles of emotional detachment and emotional sensitivity. Study 2 demonstrated that an extreme form of emotional detachment (i.e. sadism) consistently served as a partial mediator for proactive aggression, in the same way that a further set of emotions characteristic of emotional detachment (i.e. callousness, uncaring and unemotional) did so in Study 3. The findings from Study 3 showed that callous-unemotional traits associated with both proactive and reactive aggression, whereas inhibitory control problems, conceptualised in Study 2 as emotional dysregulation, related more to reactive aggression (Thomson \& Centifanti, 2018). In doing so, this lends support to the previously noted findings of Garofalo et al (2018), and also those of Long et al. (2014), 
with the latter noting how the psychopathy-impulsive (reactive) aggression relationship was mediated by emotional dysregulation.

The finding that sadism was also a partial mediator between psychopathy and reactive aggression fits with callous emotions partially mediating the relationship between psychopathy and aggression in Study 2, since callousness is particularly consistent with sadism. Thus, an emotional detachment component to the psychopathy-reactive aggression relationship cannot be fully ruled out. However, it would appear it was emotional-sensitivity/poor regulation that mediated between psychopathy and reactive aggression (Study 2), with emotional detachment important for understanding the association between psychopathy and proactive aggression (Study 3). This highlights the importance of the Violence Inhibition Mechanism (VIM: Blair, $1995,2001)$ in the psychopathy-aggression relationship, suggesting that it is the failure to respond to distress cues in others that is important: this could occur either through not recognising the distress or not caring about it, which is associated with the view that those with psychopathy fail to develop a social or moral conscience (Frick \& Morris, 2004). It further suggests value in researching areas closely aligned to sadism, such as contempt and spitefulness, which have been found associated with increased levels of psychopathy (Garofalo et al, 2019). This highlights again how the concept of emotion needs to be broadly considered when applied to the concept of psychopathy (Garofalo et al, 2018).

Collectively, the studies presented here suggest that emotions associated with emotional detachment are more important in describing the psychopathy and proactive aggression relationship, whereas the emotional-sensitivity/poor regulation aspect is important to the psychopathy-reactive aggression relationship. It could be speculated, therefore, that proactive aggression is consistent more with the atypical emotional challenges found with primary psychopathy/Factor I psychopathy, and reactive aggression more with atypical secondary psychopathy/Factor II psychopathy. The importance of avoiding viewing 
psychopathy as a homogenous concept is also clearly evidenced, particularly when considering its association with aggression (e.g. Garofalo et al, 2018; Garofalo et al, 2019) and when examining a range of emotions likely to be important (Garofalo et al, 2019). Indeed, what is clear is that a deficit in emotional experience is too limited a description of the emotional world of those with psychopathic traits (Garofalo et al, 2019).

This echoes more recent conceptualisations of psychopathy, such as De Lisi's proposal that psychopathy represents a Unified Theory of Crime (DeLisi, 2009, 2016). This argues that psychopathy is the (acute) manifestation of criminality, comprised of various affect challenges, including marked differences in how they experience their emotional world compared to those who are not presenting with psychopathy (DeLisi, 2009). The Unified Theory views psychopathy as a total concept in its own right, seeing it as the motivation for offending, of which aggression forms part. Psychopathy is not considered by this theory as a mere correlate of offending. The theory would consider psychopathy the best explanation, therefore, for aggression, particularly if this is a stable characteristic (DeLisi, 2009, 2016). What the current study is highlighting, is perhaps a mechanism through which psychopathy and certain forms of aggression motivation are presenting, with this mechanism representing emotional reactions that appear markedly different among those with psychopathic traits.

Importantly, neither set of core concepts examined in the current studies (i.e. aggression motivation and psychopathy) are considered dichotomous. Both are continuous measures, with individuals falling at some point along a continuum. A pragmatic interpretation of the current results would speculate that when psychopathic traits are functionally related to an act of aggression, if that aggression falls more towards the proactive end of the spectrum then the emotions partially driving this are likely consistent with the manifestation of primary/Factor I psychopathic traits, namely emotional detachment. If the aggression falls more towards the reactive end of the aggression spectrum, the emotions influencing it are likely to be more 
consistent with the manifestation of secondary/Factor II psychopathic traits, namely emotionalsensitivity/poor regulation.

It is further suggested that more consideration should be given to the manifestation of psychopathy traits at any given time and how these could differentially influence what promotes aggression use. Separating groups into primary/secondary psychopathy may not be as relevant as concluding that when they selected a particular form of aggression they were manifesting psychopathic traits that were consistent with and/or influenced primarily by either a lack of care and concern or hypersensitivity/poor regulation. Such an interpretation would suggest that preventing aggression demonstrated by those with a history of psychopathy would have to be sensitive to the type of aggression that was manifested and what end of the spectrum of emotions was consequently important; teaching regulation and displacement/distraction skills for the more reactive forms of aggression may be valuable, whereas for the more proactive forms, targeting consequences (either for themselves, or others), may be beneficial.

The current studies are not, however, without their limitations. They used a nonoffending sample. Although there are likely to be benefits with this when attempting to ensure that antisocial behaviour does not dominate (Kimonis et al., 2006), it is nevertheless the case that the current samples are not at the more extreme end of psychopathy, which occurs in clinical or forensic populations. The study is also reliant on self-report, which has obvious limitations, including under (and over) reporting of traits and behaviours. This is a common criticism. It is, for example, acknowledged that in the current study the very nature of psychopathic traits lends itself to challenges in the veracity of self-report. This challenge could have been managed through the use of different methods, or a combination of methods, such as informant information or collateral information. The practicalities of obtaining such information are not insignificant but self-report is, nevertheless, a limitation that needs to be acknowledged in full. It has been well recognised by other researchers engaged in similar 
research (e.g. Blais et al, 2014; Garofalo et al, 2019) and is thus not unique as a criticism. Connected to self-report, the study also used three different self-report measures of psychopathy, to demonstrate some generalisability of findings, to guard against criticism that the results could be an artefact of a particular measure of psychopathy, and to allow for psychopathy factors to be captured as well as an examination of primary/secondary psychopathy. Although adopting an approach of using different measures within a set of studies is not novel (e.g. Garofalo et al, 2019), there are limitations in consistency that are fully accepted. An additional limitation is that all the studies were cross-sectional, i.e. they did not provide an assessment of the changing experiences of emotions and/or aggression over time, or the part played by more implicit emotional processing.

Future research would benefit from capturing in more detail the dynamic and changing motivations for aggression; motivation can alter over time and change with context. However, it is more commonly considered in static, unchanging, terms within research. The same is true for the concept of psychopathy, with this increasingly being considered a more dynamic concept (Lewis et al, 2017) and, thus, a direction that future research could accommodate for. The current research demonstrates the importance of considering what impacts on the psychopathy-aggression relationship. It highlights the need to study both psychopathy and aggression as heterogeneous concepts, and points to the potential role of emotions in explaining the aggression-psychopathy relationship. Considering a range of emotions has proven important, particularly those that fall along the emotional detachment versus emotionalsensitivity/poor regulation ends of the emotional spectrum. What is of further importance, but as yet not fully considered, is the role of the environment and how this could interact with those presenting with psychopathy who are choosing to display aggression. The potential role of the environment in facilitating or inhibiting an emotional response is not yet considered in this regard. Whilst it is accepted that individual tendencies, including emotional reactions and 
responses, are important, what is being increasingly recognised is a role for the environment. Individual explanations for psychopathy are increasingly being argued to have less relevance (DeLisi, 2009. 2016) than more dynamic explanations. To date, no such explanations have been offered to explain the potential interaction between the environment and emotion as a mechanism for aggression among those with raised psychopathy traits, and how this could link to various forms of aggression and other antisocial behaviour. 
Table 1. AQ and PAPA scores overall and across subscales.

\begin{tabular}{lll}
\hline Measure & Overall M (SD/n) & Scale $\boldsymbol{\alpha}$ \\
\hline PAPA Total & $14.9(5.5 / 150)$ & .88 \\
PAPA: Dissocial & $14.9(5.5 / 150)$ & .77 \\
PAPA: Emotional detachment & $7.8(3.0 / 150)$ & .67 \\
PAPA: Disregard for others & $13.9(4.5 / 150)$ & .73 \\
PAPA: Sensitivity to negative emotion & $18.7(5.3 / 150)$ & .92 \\
AQ Total & $76.4(21.1 / 150)$ & .86 \\
AQ: Physical & $21.8(8.3 / 150)$ & .85 \\
AQ: Verbal & $14.6(3.6 / 150)$ & .86 \\
AQ: Anger & $17.9(6.9 / 150)$ & \\
\hline
\end{tabular}


Table 2. Psychopathy, proactive and reactive aggression, and emotional dysregulation overall and across sex $(n=442 ; 314 M, 123 F)$.

\begin{tabular}{lllll}
\hline Scale & Overall M & Men & Women & Scale $\boldsymbol{\alpha}$ \\
& (SD) & M (SD) & M (SD) & \\
& & & & \\
\hline SD3: Psychopathy & $21.1(5.8)$ & $21.7(5.8)$ & $19.5(5.5)$ & .79 \\
SSIS: Sadism & $11.6(1.9)$ & $11.6(1.8)$ & $11.6(1.9)$ & .82 \\
Reactive aggression & $8.4(3.9)$ & $8.1(3.7)$ & $9.0(4.3)$ & .85 \\
Proactive aggression & $2.5(2.7)$ & $2.5(2.7)$ & $2.2(2.6)$ & .89 \\
DERS: Emotional & $88.9(23.3)$ & $85.8(22.0)$ & $96.6(24.8)$ & .93 \\
[dys]regulation & & & & \\
\end{tabular}



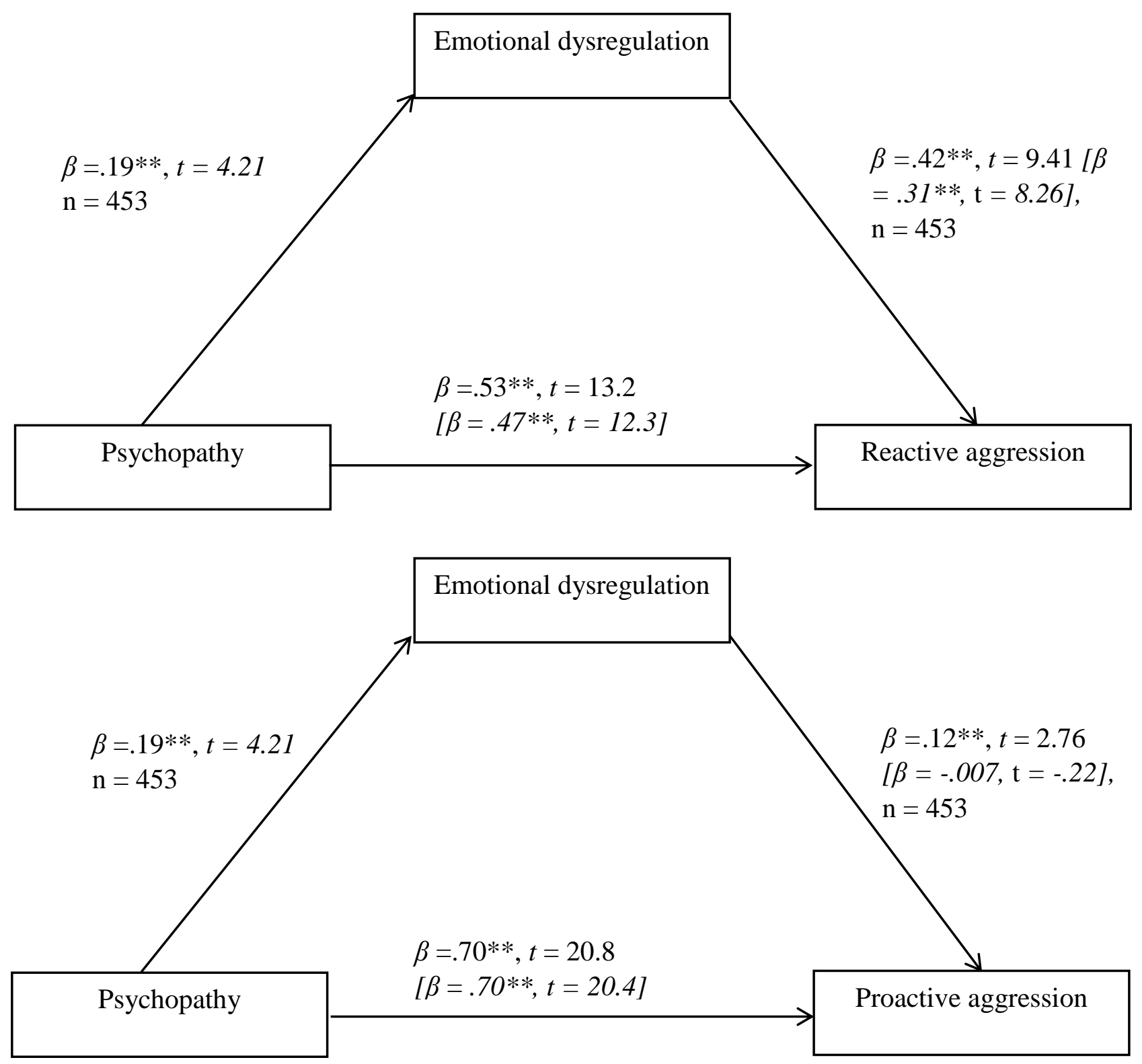

Figure 1. Standardised regression coefficients for the relationship between psychopathy and aggression as mediated by increased emotional dysregulation. The standardized regression coefficient between psychopathy and each aggression motivation, controlling for emotional dysregulation, is in parentheses. $p<.001 * *$. 


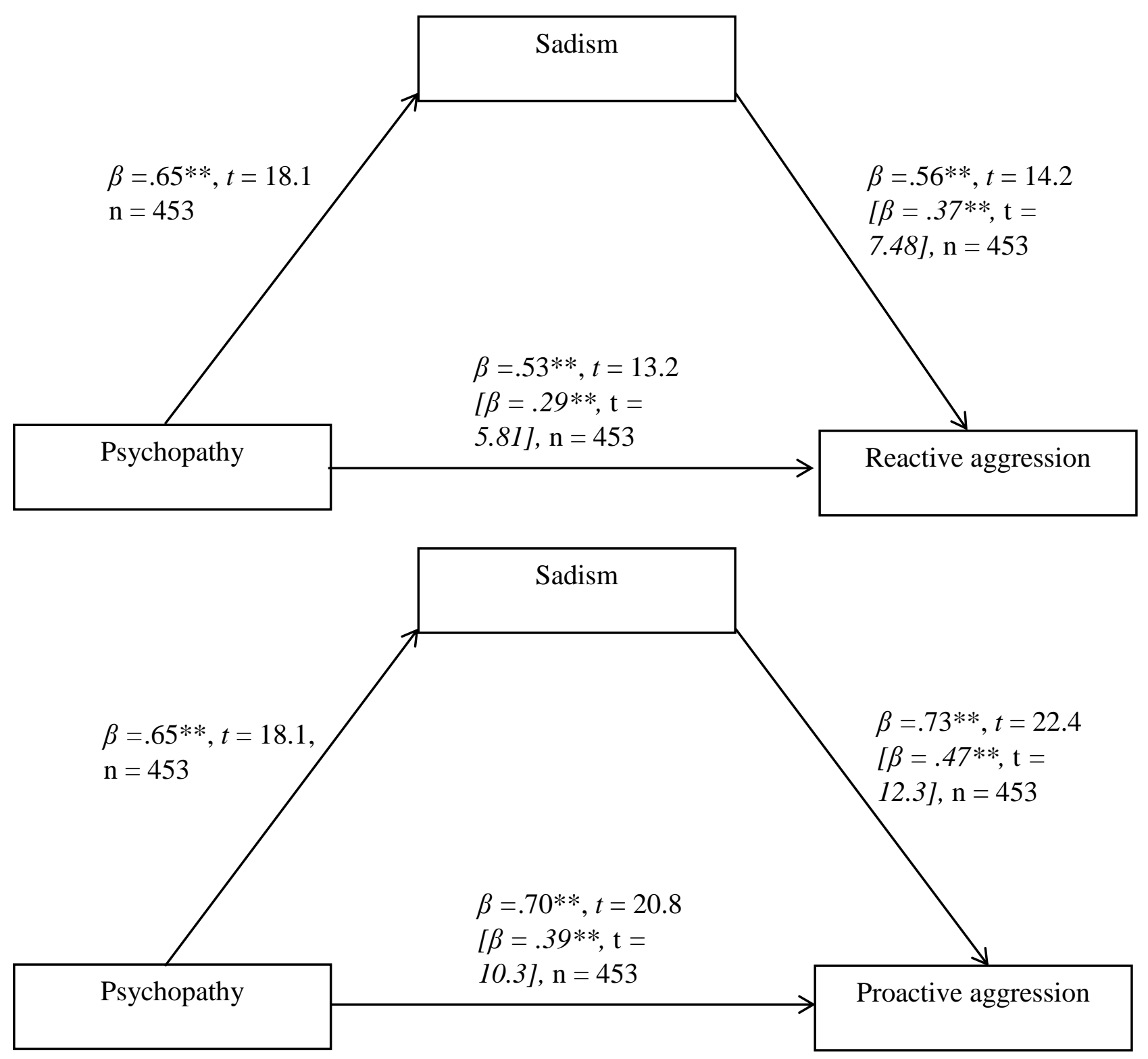

Figure 2. Standardised regression coefficients for the relationship between psychopathy and aggression as mediated by increased sadism. The Standardized regression coefficient between psychopathy and each aggression motivation, controlling for sadism, is in parentheses. $p$ $<.001 * *$. 
Table 3. Psychopathy, proactive and reactive aggression, and callous-unemotional traits overall and across sex $(n=198)$.

\begin{tabular}{llll}
\hline Measure & M & (SD) & Scale $\boldsymbol{\alpha}$ \\
\hline RPQ & 8.12 & $(4.2)$ & .84 \\
Reactive & 1.17 & $(1.02)$ & .84 \\
Proactive & & & .78 \\
\hline LSRP & 15.89 & $(8.1)$ & .78 \\
Prim psychopathy & 11.17 & $(4.3)$ & .70 \\
Secondary psychopathy & & & .75 \\
\hline ICU & & $(.83)$ & .73 \\
Callousness & 2.24 & $(3.8)$ & $(2.9)$ \\
Uncaring & 7.79 & & \\
Unemotional & 7.19 & & \\
\hline
\end{tabular}



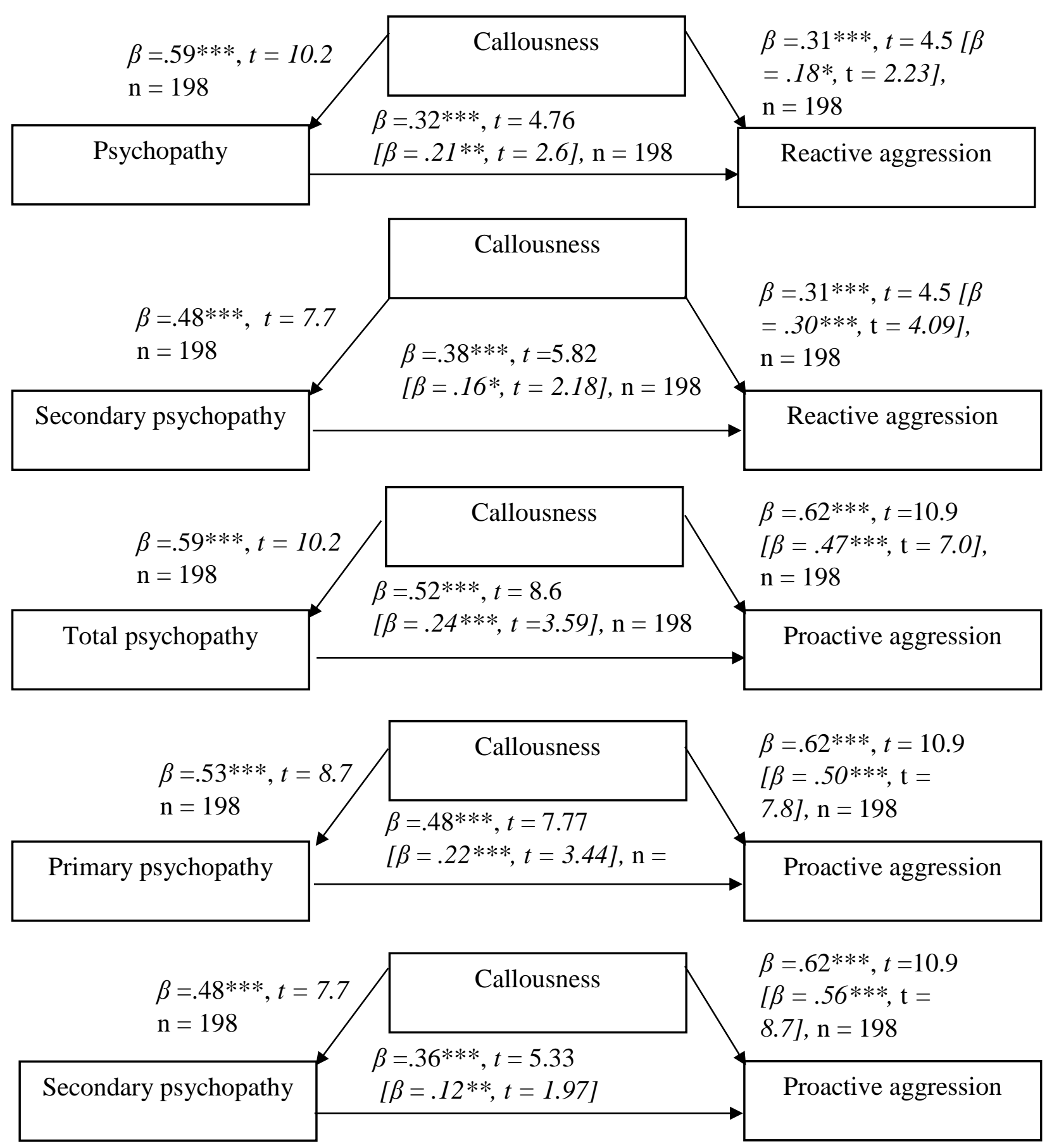

Figure 3. Standardised regression coefficients for the relationship between psychopathy and aggression as mediated by increased callousness. The standardized regression coefficient between psychopathy and each aggression motivation, controlling for callousness, is in parentheses. $p<.001 * * * ; p<.01 * * ; p<.05 *$. 

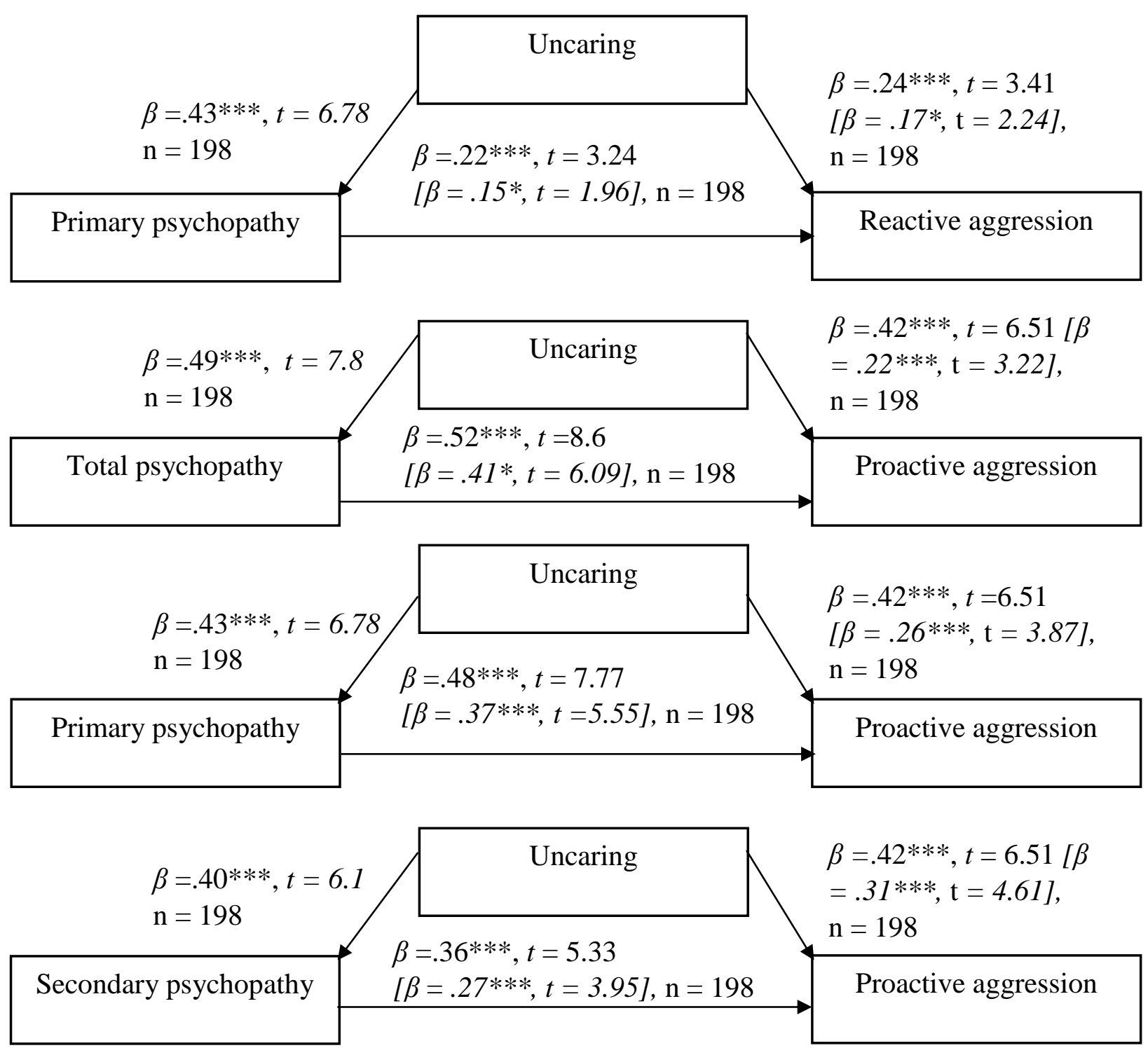

Figure 4. Standardised regression coefficients for the relationship between psychopathy and aggression as mediated by increased uncaring. The standardized regression coefficient between psychopathy and each aggression motivation, controlling for uncaring, is in parentheses. $p<.001 * * ; p<.01 * * ; p<.05 *$. 


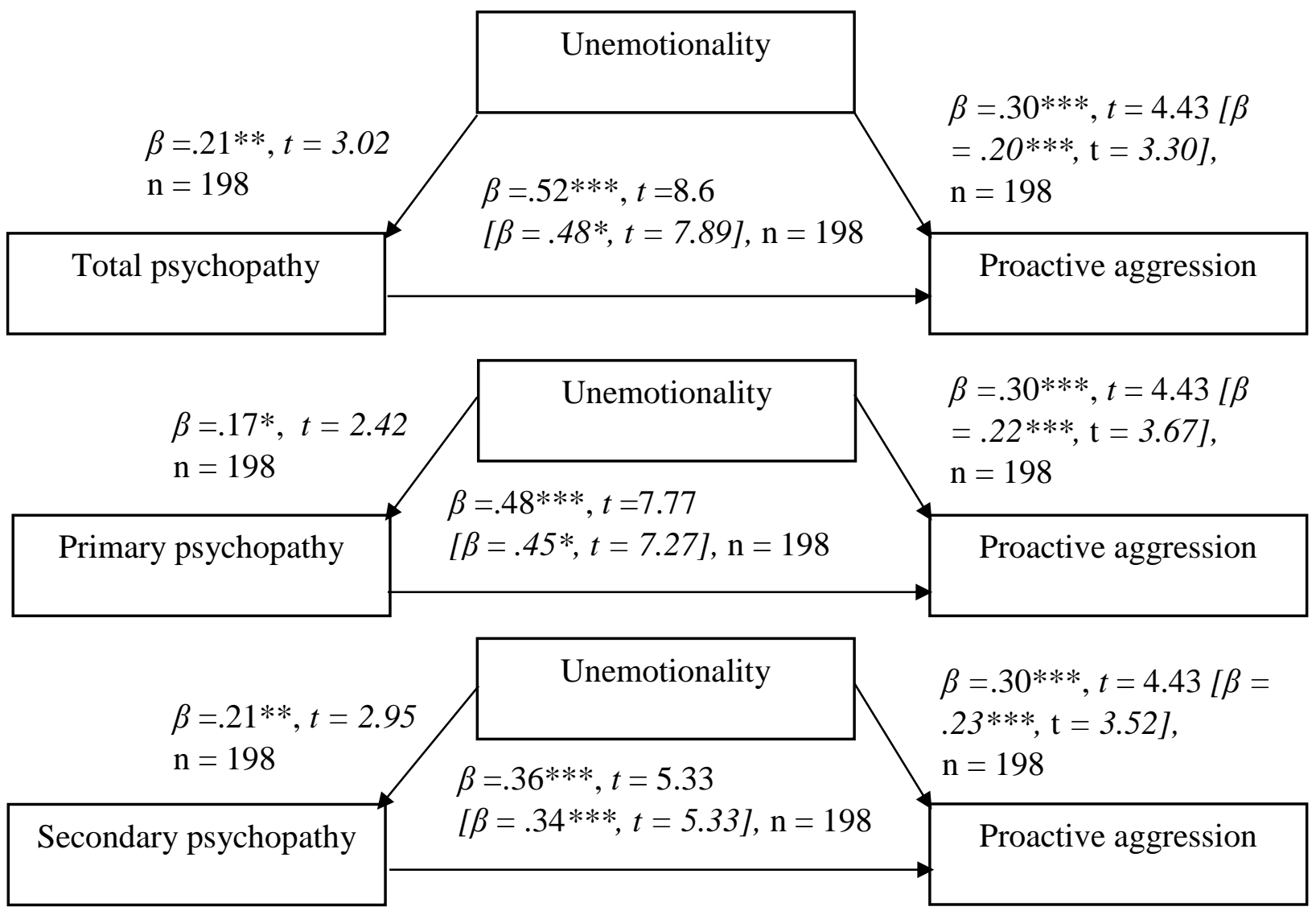

Figure 5. Standardised regression coefficients for the relationship between psychopathy and aggression as mediated by increased unemotionality. The standardized regression coefficient between psychopathy and each aggression motivation, controlling for unemotionality, is in parentheses. $p<.001 * * ; p<.01 * * ; p<.05 *$. 


\section{References}

Archer, J., \& Thanzami, V. L (2009). The relation between mate value, entitlement, physical aggression, size and strength, among a sample of young Indian men. Evolution and Human Behavior, 30, 315-321.

Asscher, J. J., van Vugt, E. S., Stams, G. J. J. M., Deković, M., Eichelsheim, V. I., \& Yousfi, S. (2011). The relationship between juvenile psychopathic traits, delinquency and (violent) recidivism: A meta-analysis. Journal of Child Psychology and Psychiatry, 52, 1134-1143.

Berg, J. M., Lilienfeld, S. O., Reddy, S. D., Latzman, R. D., Roose, A., Craighead, L. W., Pace, T. W. W., \& Raison, C. L. (2013). The Inventory of Callous and Unemotional Traits: A construct-validation analysis in an at-risk sample. Assessment, 20, 532-544.

Bezdjian, S., Tuvblad, C., Raine, A., \& Baker, L. A. (2011). The genetic and environmental covariation among psychopathic personality traits, and reactive and proactive aggression in childhood. Child Development, 82, 1267-1281.

Blackburn, R. (1998). Psychopathy and personality disorder: Implications of Interpersonal

Theory. In D. J. Cooke., A. E. Forth., \& R. D., Hare (Eds.). Psychopathy: Theory, research and implications for society (pp. 269-301). Dordrecht, The Netherlands: Kluwer.

Blair, R. J. R. (1995). A cognitive developmental approach to morality: Investigating the psychopath. Cognition, 57, 1-29.

Blair, R. J. R. (2001). Neurocognitive models of aggression, the antisocial personality disorders and psychopathy. Journal of Neurology, Neurosurgery, and Psychiatry, 71, 727-731.

Blair, R. J. R., Morris, J. S., Frith, C. D., Perrett, D. I., \& Dolan, R. J. (1999). Dissociable neural responses to facial expressions of sadness and anger. Brain, 122, 883-893. 
Blais, J., Solodukhin, E., \& Forth, A. E. (2014). A meta-analysis exploring the relationship between psychopathy and instrumental versus reactive violence. Criminal Justice and Behavior, 41, 797-821.

Brugman, S., Cornet, L. J. M., Smeijers, D., Smeets, K., Oostermeijer, S., Buitelaar, J.K., ... Jansen, L. M. C. (2017). Examining the Reactive Proactive Questionnaire in Adults in forensic and non-forensic settings: A variable- and person-based approach. Aggressive Behavior, 43, 155-162.

Buss, A. H., \& Perry, M. (1992). The Aggression Questionnaire. Journal of Personality and Social Psychology, 63, 452-459.

Cima, M., Raine, A., Meesters, C., \& Popma, A. (2013). Validation of the Dutch Reactive Proactive Questionnaire (RPQ): Differential correlates of reactive and proactive aggression from childhood to adulthood. Aggressive Behavior, 39, 99-113.

Cornell, D. G, Warren, J., Hawk, G., Stafford, E., Oram, G., \& Pine., D. (1996). Psychopathy in instrumental and reactive violent offenders. Journal of Consulting and Clinical Psychology, 64, 783-790.

DeLisi, M. (2016). Psychopathy as Unified Theory of Crime. Palgrave Macmillan, New York.

DeLisi, M. (2009). Psychopathy is the unified theory of crime. Youth Violence and Juvenile Justice, 7, 256.

Domes, G., Hollerbach, P., Vohs, K., Mokros, A., \& Habermeyer, E. (2013). Emotional empathy and psychopathy in offenders: An experimental study. Journal of Personality Disorders, 27, 67-84.

Fido, D., Santo, M. G. E., Bloxsom, C. A. J., Gregson, M., \& Sumich, A. L. (2017). Electrophysiological study of the violence inhibition mechanism in relation to callousunemotional and aggressive traits. Personality and Individual Differences, 118, 44-49. 
Frick, P. J. (2004). The Inventory of Callous-unemotional Traits. Unpublished rating scale: University of New Orleans.

Frick, P. J., \& Morris, A. S. (2004). Temperament and developmental pathways to conduct problems. Journal of Clinical Child and Adolescent Psychology, 33, 54-68.

Garofalo, C., Neumann, C. S., \& Velotti, P. (2018). Difficulties in emotion regulation and psychopathic traits in violent offenders. Journal of Criminal Justice, 57, 116-125.

Garofalo, C., Neumann, C. S., Zeigler-Hill, V., \& Meloy, J. R. (2019). Spiteful and contemptuous: A new look at the emotional experiences related to psychopathy. Personality Disorders: Theory, Research, and Treatment, 10, 173.

Gratz, K. L., \& Roemer, L. (2004). Multidimensional assessment of emotion regulation and dysregulation: Development, factor structure, and initial validation of the difficulties in emotion regulation scale. Journal of Psychopathology and Behavioral Assessment, 26, $41-54$.

Guy, L. S., Douglas, K. S., \& Hendry, M. C. (2010). The role of psychopathic personality disorder in violence risk assessments using the HCR-20. Journal of Personality Disorders, 24, 551-580.

Hare, R.D. (2003). Manual for the revised Psychopathy Checklist Revised 2. Multi-Health Systems. Toronto, ON: Canada.

Ireland, J. L., Mann, S., Lewis, M., Ozanne, R., McNeill, K., \& Ireland, C. A. (under review). Psychopathy and trauma: Exploring a potential association.

Jones, D. N., \& Paulhus, D. L. (2014). Introducing the Short Dark Triad (SD3). Assessment, $21,28-41$.

Kimonis, E. R., Frick, P. J., Fazekas, H., \& Loney, B. R. (2006). Psychopathy, aggression and the processing of emotional stimuli in non-referred girls and boys. Behavioural Sciences and the Law, 24, 21-37. 
Levenson, M. R., Kiehl, K. A., \& Fitzpatrick, C. M. (1995). Assessing psychopathic attributes in a noninstitutionalized population. Journal of Personality and Social Psychology, 68, $151-158$.

Lewis, M. (2014). Understanding the affective and cognitive components of psychopathy: Developing a new assessment. Doctoral dissertation. University of Central Lancashire, Preston, UK.

Lewis, M., Ireland, J. L., Abbott, J., \& Ireland, C. A. (2017). Initial development of the Psychopathic Processing and Personality Assessment (PAPA) across populations. International Journal of Law and Psychiatry, 54, 118-132.

Lewis, M., Ireland, J. L., Ireland, C. A, Derefaka, G., McNeill, K., Birch, P., et al. (under review). Psychopathic Processing and Personality Assessment (PAPA): Exploring factor structure.

Loney, B. R., Frick, P. J., Clements, C. B., Ellis, M. L., \& Kerlin, K. (2003). Callousunemotional traits, impulsivity, and emotional processing in adolescents with antisocial behavior problems. Journal of Clinical Child and Adolescent Psychology, 32, 66-80.

Long, K., Felton, J. W., Lilienfeld, S. O., Lejuez, C. W. (2014). The role of emotion regulation in the relations between psychopathy factors and impulsive and premeditated aggression. Personality Disorders: Theory, Research, and Treatment, 5, 390-396.

Ohlsson, I. M., \& Ireland, J. L. (2011). Aggression and offence motivation in prisoners: Exploring the components of motivation in an adult male sample. Aggressive Behavior, 37, 278-288.

O’Meara, A., Davies, J., \& Hammond, S. (2011). The psychometric properties and utility of the Short Sadistic Impulse Scale (SSIS). Psychological Assessment, 23, 523-531.

Porter, S., \& Woodworth, M. (2006). Psychopathy and aggression. In C. Patrick (Ed.). Handbook of psychopathy (pp. 481-494). New York: Guildford Press. 
Raine, A., Dodge, K., Loeber, R., Gatze-Kopp, L., Lynam, D., Reynolds, C., StouthamerLoeber, M., \& Liu, J. (2006). The Reactive-Proactive Aggression Questionnaire: Differential correlates of reactive and proactive aggression in adolescent boys. Aggressive Behavior, 32, 159-171.

Raine., A., Fung A. L., Portnoy, J., Choy, O., \& Spring, V. L. (2014). Low heart rate as a risk factor for children and adolescent proactive aggression an impulsive psychopathic behaviour. Aggressive Behaviour, 40, 290-299.

Roberton, T., Daffern., M., Bucks., R. S. (2012). Emotional regulation and aggression. Aggression and Violent Behavior, 17, 72-82.

Thomson, N. D., \& Centifanti, L. C. M. (2018). Proactive and reactive aggression subgroups in typically developing children: The role of executive functioning, psychophysiology, and psychopathy. Child Psychiatry and Human Development, 49, 197-208.

Thomson, N. (2019). Understanding Psychopathy: The Biopsychosocial Perspective. London: Routledge.

Vitacco, M. J., Neumann, C. S., \& Caldwell, M. F. (2010). Predicting antisocial behavior in high-risk male adolescents. Criminal Justice and Behavior, 37, 833-846.

Vitaro, F \& Brendgen, M. (2005). Proactive and reactive aggression. In R. Tremblay, W.W. Hartup, \& J. Archer (Eds.), Developmental Origins of Aggression (pp. 178-201). New York: Guilford.

Walsh, Z., Swogger, M. T., \& Kosson, D. S. (2009). Psychopathy and instrumental violence: Facet level relationships. Journal of Personality Disorders, 23, 416-424.

Walters, G. D. (2003). Predicting institutional adjustment and recidivism with the Psychopathy Checklist factor scores: A meta-analysis. Law and Human Behavior, 27, $541-558$. 